\title{
Topological Entropy of One Type of Nonoriented Lorenz-Type Maps
}

\author{
Guo Feng \\ Basic Subject Department, Shandong Women’s University, Jinan 250300, China \\ Correspondence should be addressed to Guo Feng; sdwugf@163.com
}

Received 16 June 2016; Accepted 29 September 2016

Academic Editor: Massimiliano Ferrara

Copyright (c) 2016 Guo Feng. This is an open access article distributed under the Creative Commons Attribution License, which permits unrestricted use, distribution, and reproduction in any medium, provided the original work is properly cited.

Constructing a Poincaré map is a method that is often used to study high-dimensional dynamical systems. In this paper, a geometric model of nonoriented Lorenz-type attractor is studied using this method, and its dynamical property is described. The topological entropy of one-dimensional nonoriented Lorenz-type maps is also computed in terms of their kneading sequences.

\section{Introduction}

The Lorenz attractor is usually divided into three types, which are called oriented, semioriented, and nonoriented Lorenz attractor, respectively, and the existence condition of Lorenz attractor of planar map is given in [1]. The oriented dynamical property is studied in detail in [2-4]. In this paper, the nonoriented situation is discussed. Lorenz system is approximated by the Shimizu-Morioka model $(\dot{x}=y$, $\dot{y}=x-a y-x z, \dot{z}=-b z+x^{2}$ ) when the parameter $r$ is large. This model has nonoriented Lorenz attractors for certain parameters, (e.g., $a \approx 0.59$ and $b \approx 0.45$ ). The bifurcations and chaos of the model are discussed using numerical method in $[5,6]$. In this paper, a geometric model of the type attractor is described, and a formula for computation of the topological entropy of one-dimensional nonoriented Lorenz maps is given.

\section{A Geometric Model of the Nonoriented Lorenz-Type Attractors}

The differential equation with a single parameter $\dot{X}=$ $f(\mu, X), X \in R^{3}$, is considered, and it is symmetric about $z$ axis. The butterfly homoclinic orbit exists when the parameter $\mu$ is zero and the equilibrium point is $O=(0,0,0)$. The eigenvalue of the linearization matrix at $O$ satisfies $0<c \leq a<b$. Through simple coordinate transformations, the equation can be reduced to

$$
\begin{aligned}
& \dot{x}=a x+P(\mu, x, y, z) \\
& \dot{y}=-b y+Q(\mu, x, y, z) \\
& \dot{z}=-c z+R(\mu, x, y, z),
\end{aligned}
$$

where $P, Q$, and $R$ are high-order items. In the neighborhood of $O$, the dynamical property of the equations can be described by its linear part. Due to the symmetry of the system, we only discuss the situation $x \geq 0$. The sections $\Sigma_{0}: z=d$ and $\Sigma_{1}: x=d$ (where $d>0$ ) are taken near the equilibrium $O$. The sections are shown in Figure 1(a). The solution of the linear equations $\dot{x}=a x, \dot{y}=-b y$, and $\dot{z}=-c z$ are $x=x_{0} e^{a t}, y=y_{0} e^{-b t}$, and $z=d e^{-c t}$ with the initial point $\left(x_{0}, y_{0}, d\right)$ on $\Sigma_{0}$. The time of the flow with the initial point from $\Sigma_{0}$ to $\Sigma_{1}$ is $\tau=(1 / a) \ln \left(d / x_{0}\right)$ with $d=x_{0} e^{a \tau}$. Then the map $T_{0}: \Sigma_{0} \rightarrow \Sigma_{1} T_{0}$ is defined by

$$
\begin{aligned}
& y_{1}=y_{0} e^{-b \tau} \\
& z_{1}=d e^{-c \tau} ;
\end{aligned}
$$

that is,

$$
\begin{aligned}
& y_{1}=y_{0} x_{0}^{\gamma} d^{-\gamma} \\
& z_{1}=x_{0}^{\nu} d^{1-\nu},
\end{aligned}
$$




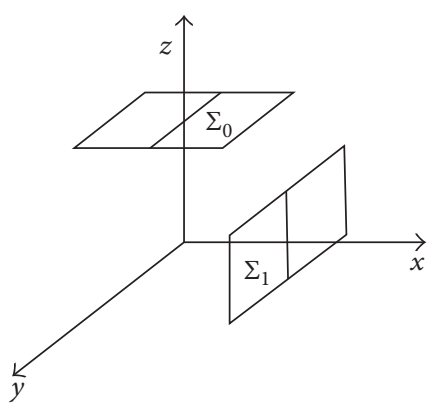

(a)

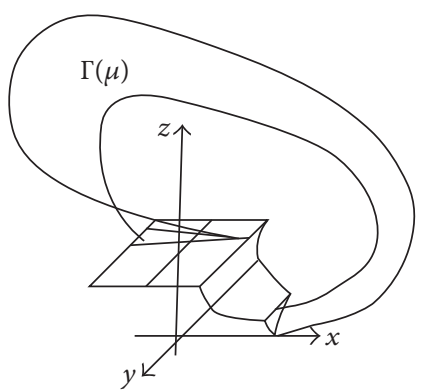

(b)

Figure 1: Poincaré map of the Lorenz.

where $\nu=c / a$ is called the saddle point index and $\gamma=b / a$. Let $\Gamma(0)$ be the homoclinic orbit of the unperturbed system (when $\mu=0$ ), and let $\Gamma(\mu)$ be its perturbed solution; see Figure 1(b). Then the flows adjacent $\Gamma(\mu)$ determine a map $T_{1}: \Sigma_{1} \rightarrow \Sigma_{0}$. In the small neighborhood of the origin $M$ on $\Sigma_{1}$, the map $T_{1}: \Sigma_{1} \rightarrow \Sigma_{0}$ can be written as

$$
\begin{aligned}
& \bar{x}_{0}=\mu+a_{11} z_{1}+a_{12} y_{1}+\cdots \\
& \bar{y}_{0}=u(\mu)+a_{21} z_{1}+a_{22} y_{1}+\cdots .
\end{aligned}
$$

When the determinant of a matrix $\left(\begin{array}{lll}a_{11} & a_{12} \\ a_{21} & a_{22}\end{array}\right)$ is less than zero, the manifold by the map $T_{1}$ defined is nonoriented. $\mu$ is a splitting parameter, and the coordinate of $M_{0}(\mu)=\Gamma(\mu) \cap \Sigma_{0}$ is $(\mu, u(\mu))$. The map $T=T_{1} \circ T_{0}$ is obtained on $\Sigma_{0}$ :

$$
\begin{aligned}
& \bar{x}_{0}=\mu+a_{11} d^{1-\gamma} x_{0}^{\nu}+a_{12} d^{-\gamma} y_{0} x_{0}^{\gamma}+\cdots \\
& \bar{y}_{0}=u(\mu)+a_{21} d^{1-\gamma} x_{0}^{\gamma}+a_{22} d^{-\gamma} y_{0} x_{0}^{\gamma}+\cdots .
\end{aligned}
$$

Let $d=1$ by scaling variable, and remove the under index zero. Thus, we can obtain

$$
\begin{aligned}
& \bar{x}=\mu+A x^{\nu}+a_{12} y x^{\gamma}+\cdots \\
& \bar{y}=u(\mu)+a_{21} x^{\nu}+a_{22} y x^{\gamma}+\cdots,
\end{aligned}
$$

where $A=a_{11}$ is called the boundary line. This paper discusses the nonoriented Lorenz-type map corresponding to the situation $A<0$ and $\mu>0$, and let $a_{12}, a_{21}$, and $a_{22}$ be all greater than zero.

Because the system is symmetric, we consider the case of $x \leq 0$ and obtain the map

$$
\begin{aligned}
& \bar{x}=\left(\mu+A|x|^{\nu}+a_{12} y|x|^{\gamma}+\cdots\right) \operatorname{sign}(x) \\
& \bar{y}=\left(u(\mu)+a_{21}|x|^{\nu}+a_{22} y|x|^{\gamma}+\cdots\right) \operatorname{sign}(x) .
\end{aligned}
$$

Considering the truncated map,

$$
\begin{aligned}
& \bar{x}=\left(\mu+A|x|^{\nu}\right) \operatorname{sign}(x) \\
& \bar{y}=\left(u(\mu)+a_{21}|x|^{\nu}+a_{22} y|x|^{\gamma}\right) \operatorname{sign}(x) .
\end{aligned}
$$

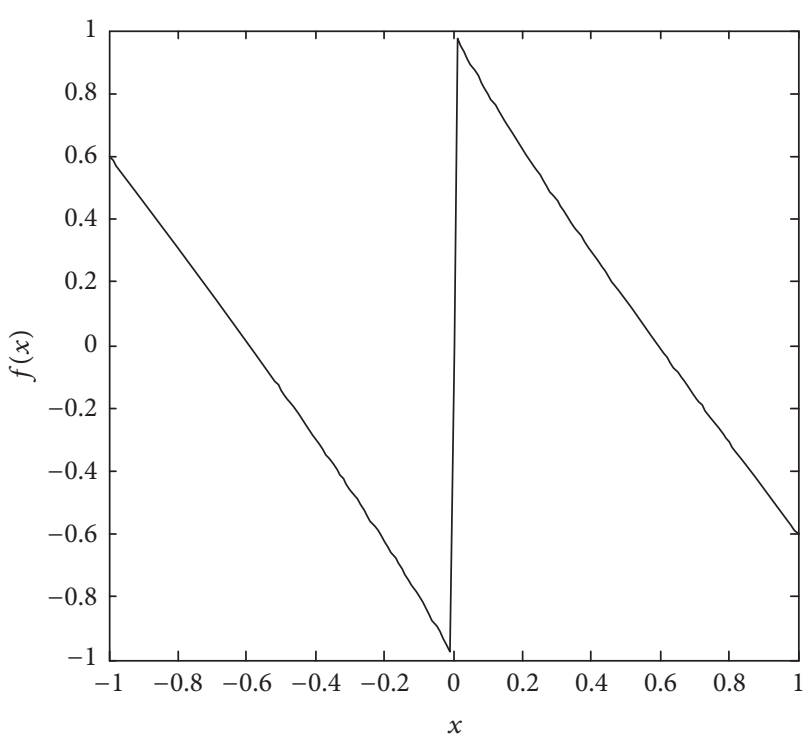

FIGURE 2: Graph of $f(x)$ while $\mu=1, A=-1.6$, and $\nu=0.9$.

The above formula is the Lorenz Poincaré map as shown in Figure 1(b).

For simplicity, we write the above map as $(\bar{x}, \bar{y})=$ $F(x, y)=(f(x), g(x, y))$. Taking $\mu=1, u(\mu)=0.5$, and $U=(-1 \leq x \leq 1,-1 \leq y \leq 1)$ on $\Sigma_{0}$, as $|1+A|<1$, $\left|0.5+a_{21}+a_{22}\right|<1$. The graph of $f(x)$ is shown in Figure 2. The region $U$ is the contraction region of the map $F(x, y)$ as shown in Figure 3.

It is similar to the condition of an existing strange attractor about oriented Lorenz map in [2]. When $|\partial f / \partial x|>$ $\sqrt{2},|\partial g / \partial y|<1 / 2$; that is, $A \nu>\sqrt{2}$ and $a_{22} \gamma<1 / 2$; the invariant set $\bigcap_{n \geq 0} T^{n}(U)=\Lambda$ is a nonoriented Lorenz attractor as shown in Figure 4. Obviously, if $\mu \leq 1$, then $|\partial f / \partial x|>\sqrt{2}$ in the set $[-1,1] \backslash\{0\}$.

Being similar to [2], the dynamical property of $\left.T\right|_{\Lambda}$ can be described by the shift map of the inverse limit pace of the map $\left.f\right|_{I}$. So it is very important to study the dynamic behavior of this kind of attractor by studying the one-dimensional map $f(x)$. 


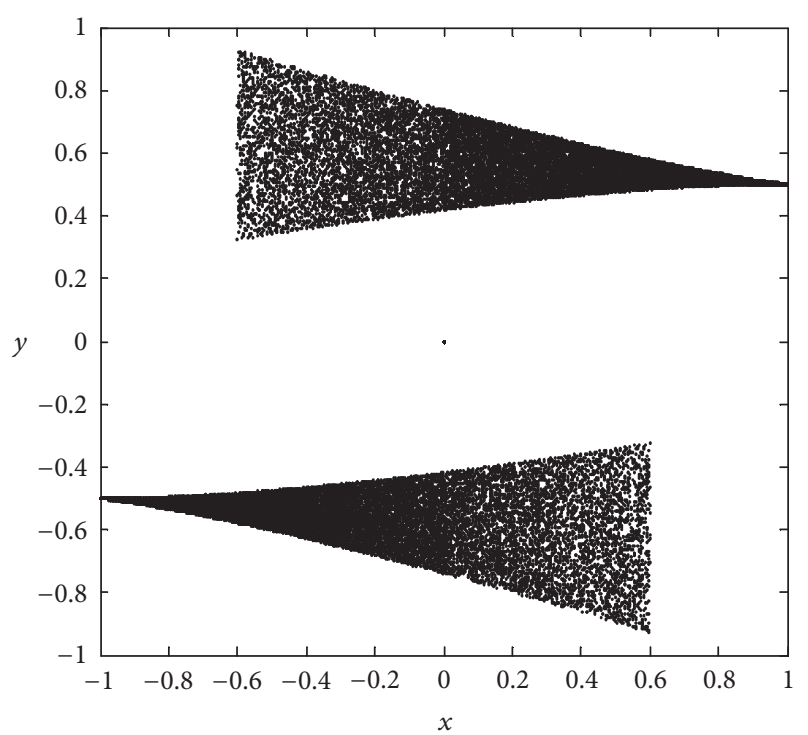

FIgURE 3: Graph of $F(U)$ while $\mu=1, u(\mu)=0.5, A=-1.6, \nu=0.9$, $a_{21}=0.1, a_{22}=0.3$, and $\gamma=1.2$.

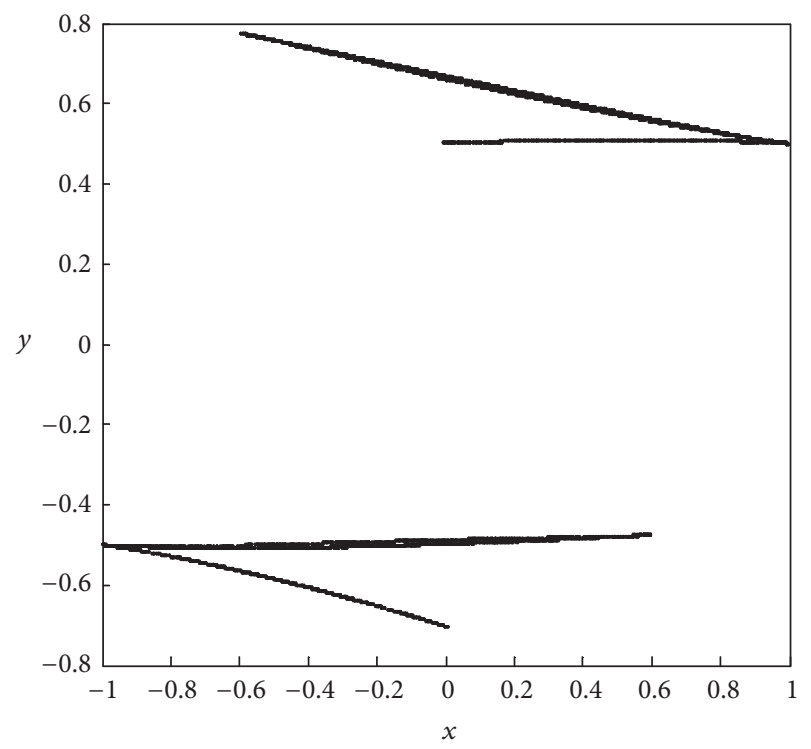

FIGURE 4: The strange attractor of $F(x, y)$ (the parameters are the same as Figure 3).

\section{The Calculation of the Topological Entropy of Nonoriented Lorenz-Type Map}

As $I=[-1,1]$, if the map $f(x): I \rightarrow I$ satisfies the condition where

(1) $[-1,0)$ and $(0,1]$ are continuous and monotone,

(2) $\lim _{x \rightarrow 0_{-}} f(x)=-1$ and $\lim _{x \rightarrow 0+} f(x)=1, f(0)=0$,

(3) the set $D(f)=\bigcup_{n \geq 0} f^{-n}(0)$ is dense on the interval $I$, then $f(x): I \rightarrow I$ is called a nonoriented Lorenz-type map.
The topological entropy of one-dimensional Lorenz-type map is defined in [7].

$$
h_{f}=\lim _{n \rightarrow \infty} \ln \frac{N_{n}}{n}=\lim _{n \rightarrow \infty} \ln \sqrt[n]{N_{n}}
$$

where $N_{n}$ is the number of discontinuous points of $f^{n}(x)$. The definition in (9) is similar to the one about piecewise monotone continuous map given by de Melo and van Strien [8]. Here, the discontinuous points correspond to the critical points in [8]. This paper will calculate topological entropy of the map $f$. In order to facilitate the calculation, for a sequence $N_{1}, N_{2}, N_{3}, \ldots$, we consider the power series

$$
N(t)=N_{1}+N_{2} t+N_{3} t^{2}+\cdots,
$$

where $t \in C$.

Lemma 1. As $n \rightarrow \infty$, the sequence $\sqrt[n]{N_{n}}=\sqrt[n]{N\left(f^{n}\right)}$ is convergent.

Proof. Firstly, we show that

$$
N(g \circ f) \leq N(g) N(f),
$$

where $f$ and $g$ are Lorenz-type maps; $N(f)$ is the number of discontinuous points of $f$. Because, in each interval on which $f$ is continuous, the number of the discontinuous points of $g$ is at most $N(g)$, the inequality $N(g \circ f) \leq N(g) N(f)$ holds. Let $k$ be a fixed natural number; for $\forall n \in \mathbf{Z}, \exists p, q \in \mathbf{Z}$ such that $n=p k+q$, where $0 \leq q<k$. By the formula,

$$
N\left(f^{n}\right) \leq\left(N\left(f^{k}\right)\right)^{p} N\left(f^{q}\right),
$$

we have

$$
\left(N\left(f^{n}\right)\right)^{1 / n} \leq\left(N\left(f^{k}\right)\right)^{p / p k+q} N(f)^{p / p k+q} .
$$

If $n \rightarrow \infty$, then $p \rightarrow \infty$, and $q$ is bounded. Thus, we have $p /(p k+q) \rightarrow 1 / k$ and $p /(p k+q) \rightarrow 0$. Therefore,

$$
\lim _{n \rightarrow \infty} \sup \left(\left(N\left(f^{n}\right)\right)^{1 / n}\right) \leq\left(N\left(f^{k}\right)\right)^{1 / k} .
$$

Since $k$ is arbitrary,

$$
\begin{aligned}
& \lim _{n \rightarrow \infty} \sup \left(\left(N\left(f^{n}\right)\right)^{1 / n}\right) \leq \inf \left(\left(N\left(f^{k}\right)\right)^{1 / k}\right) \\
& \quad \leq \lim _{k \rightarrow \infty} \inf \left(\left(N\left(f^{k}\right)\right)^{1 / k}\right)
\end{aligned}
$$

we can prove the sequence $\sqrt[n]{N_{n}}$ is convergent.

Let $\widehat{N}_{n}$ be the number of points which fall at the discontinuous points firstly when $f^{n}\left(x_{0}\right)=0$ and $f^{k}\left(x_{0}\right) \neq 0$, $k=0,1, \ldots, n-1$.

Lemma 2. $\lim _{n \rightarrow \infty} \sqrt[n]{\bar{N}_{n}}=\lim _{n \rightarrow \infty} \sqrt[n]{N_{n}}$. 
Proof. It is clear that

$$
\widehat{N}_{n} \leq N_{n} \leq n \widehat{N}_{n}
$$

and that $\lim _{n \rightarrow \infty} \sqrt[n]{N_{n}}$ exists; this also holds for $\lim _{n \rightarrow \infty} \sqrt[n]{\widehat{\bar{N}}_{n}}$. Besides, $\lim _{n \rightarrow \infty} \sqrt[n]{\widehat{N}_{n}}=\lim _{n \rightarrow \infty} \sqrt[n]{n \widehat{N}_{n}}$. Thus, we have $\lim _{n \rightarrow \infty} \sqrt[n]{\widehat{\bar{N}}_{n}}=\lim _{n \rightarrow \infty} \sqrt[n]{N_{n}}$

Let $\widehat{N}(t)=\widehat{N}_{1}+\widehat{N}_{2} t+\widehat{N}_{3} t^{2}+\cdots$ denote the power series associated with the sequence $N_{1}, N_{2}, N_{3}, \ldots$ By Lemma 2, the convergence radius of the series $1+t \widehat{N}(t)$ and $1+t N(t)$ is equal to $\lim _{n \rightarrow \infty} 1 / \sqrt[n]{\widehat{N}_{n}}=\lim _{n \rightarrow \infty} 1 / \sqrt[n]{N_{n}}$.

Now, we give the symbolic dynamics model of the onedimensional nonoriented Lorenz maps. For $x \in I$, Let $\varphi(x)=$ $\varphi_{0} \varphi_{1} \varphi_{2} \cdots$ denote its symbol sequence, where

$$
\varphi_{n}= \begin{cases}-1 & f^{n}(x)<0, \\ 0 & f^{n}(x)=0, \\ 1 & f^{n}(x)>0 .\end{cases}
$$

There is also a sequence $\varepsilon(x)=\varepsilon_{0} \varepsilon_{1} \varepsilon_{2} \cdots$, where

$$
\varepsilon_{n}= \begin{cases}-1, & \text { if } f^{n}(x) \neq 0, \\ 0, & \text { if } f^{n}(x)=0\end{cases}
$$

Thus, we can obtain a sequence

$$
k(x)=k_{0} k_{1} k_{2} \cdots,
$$

which is called the invariant coordinates of $x$, where

$$
\begin{gathered}
k_{0}=\varphi_{0}, \\
k_{1}=\varepsilon_{0} \varphi_{1}, \\
k_{2}=\varepsilon_{0} \varepsilon_{1} \varphi_{2}, \\
\vdots
\end{gathered}
$$

Therefore, we obtain a map $k: I \rightarrow \Omega_{3}^{+}$, where $\Omega_{3}^{+}$is a onesided symbol space associated with the symbols $-1,0$, and 1 . It is clear that $k(f(x))=\sigma(k(x))$, where $\sigma: \Omega_{3}^{+} \rightarrow \Omega_{3}^{+}$is the shift map. The order of invariant coordinates is as follows: if $k_{0}=k_{0}^{\prime}, \ldots, k_{n-1}=k_{n-1}^{\prime}, k_{n}<k_{n}^{\prime}$, then $k<k^{\prime}$.

Lemma 3. For $x, y \in I$, if $x<y$, then $k(x) \leq k(y)$; that is, $k: I \rightarrow \Omega_{3}^{+}$is a monotone increasing function on the interval $I$.

Proof. If $x$ and $y$ lie in the left-hand side and right-hand side of the discontinuous point 0 , respectively, then the conclusion is obvious. Suppose that $k_{k}(x)=k_{k}(y)$ for $k<n$. Let $J$ be the interval $[x, y]$. Then there is $f^{n}(x)<f^{n}(y)$ or $f^{n}(x)>f^{n}(y)$ determined by increasing or decreasing of $\left.f^{n}\right|_{J}$. When $k \leq n$, $J$ is a monotone interval of $f^{k}$. For $z \in J$, by the chain rule, $\operatorname{sign}\left(D f^{n}(z)\right)=\varepsilon_{0} \varepsilon_{1} \cdots \varepsilon_{n-1}$. If $\varepsilon_{0} \varepsilon_{1} \cdots \varepsilon_{n-1}>0$, then $f^{n}(x)<$ $f^{n}(y)$; that is, $\varphi_{n}(x)<\varphi_{n}(y)$.
If $\varepsilon_{0} \varepsilon_{1} \cdots \varepsilon_{n-1}<0$, then $f^{n}(x)>f^{n}(y)$; that is, $\varphi_{n}(x)>$ $\varphi_{n}(y)$. For both cases, we have

$$
\begin{aligned}
k_{n}(x) & =\varepsilon_{0} \varepsilon_{1} \cdots \varepsilon_{n-1} \varphi\left(f^{n}(x)\right) \\
& \leq \varepsilon_{0} \varepsilon_{1} \cdots \varepsilon_{n-1} \varphi\left(f^{n}(y)\right)=k_{n}(y),
\end{aligned}
$$

when $\varphi_{n}(x)=\varphi_{n}(y)=0$; the above equal signs hold. Obviously, if $x, y \in I \backslash D$, then $k(x)<k(y)$. Let $x+$ and $x-$ denote the left and right limit points of $x$, respectively. By Lemma $1, k(x-)$ and $k(x+)$ exist. For discussion, the sequence of point $x$ is signed by a power series in complex field; that is, $k(x, t)=k_{0}+k_{1} t+k_{2} t^{2}+\cdots$.

Lemma 4. $k(1-, t)-k(-1+, t)=\sum_{x \in \operatorname{in}(I)}[k(x+, t)-k(x-, t)]$.

Proof. The function $k(x, t) \bmod \left(t^{n}\right)$ is the series $k(x, t)$, which is to be truncated from $t^{n}$. For fixed $t, k(x, t) \bmod \left(t^{n}\right)$ is a step function with a finite number of discontinuous points; then the statement of the lemma follows.

We call $\alpha=k\left(0_{-}\right)=\lim _{x \rightarrow 0_{-}} k(x)$ and $\beta=k(0+)=$ $\lim _{x \rightarrow 0+} k(x)$ the kneading sequences of the map $f$. The result of Lemma 4 can be simplified by kneading sequences.

Theorem 5. $k(1-, t)-k(-1+, t)=\widehat{N}(t)[\beta(t)-\alpha(t)]$.

Proof. If $f^{n}\left(x_{0}\right)=0$ and $f^{k}\left(x_{0}\right) \neq 0, k=0,1, \ldots, n-1$, then

$$
\begin{aligned}
& k\left(x_{0}+, t\right)=k\left(x_{0}, t\right)+t^{n} \beta(t), \\
& k\left(x_{0}-, t\right)=k\left(x_{0}, t\right)+t^{n} \alpha(t) .
\end{aligned}
$$

Substituting the above two equations into Lemma 4, we can obtain

$$
k(1-, t)-k(-1+, t)=\sum_{n=0}^{\infty} \widehat{N}_{n} t^{n}[\beta(t)-\alpha(t)] .
$$

For one-dimensional nonoriented Lorenz-type maps,

$$
\begin{aligned}
& \sigma(\beta)=k(1-), \\
& \sigma(\alpha)=k(-1+) ;
\end{aligned}
$$

that is,

$$
\begin{aligned}
& \beta(t)=1-k(1-, t) t, \\
& \alpha(t)=-1-k(-1+, t) t .
\end{aligned}
$$

Substituting (24) and (25) into (23),

$$
[\beta(t)-\alpha(t)][1+t \widehat{N}(t)]=2 .
$$

Because the series $1+t \widehat{N}(t)$ is absolutely convergent, when $|t|<\lim _{n \rightarrow \infty} 1 / \sqrt[n]{\widehat{N}_{n}}$, the series $1+t \widehat{N}(t)$ is an analytic function in convergent circle $|t|=\lim _{n \rightarrow \infty} 1 / \sqrt[n]{\widehat{N}_{n}}$.

The series $1+t \widehat{N}(t)$ has poles in convergent circle. The series of $1+t \widehat{N}(t)$ is positive, so $|1+t \widehat{N}(t)| \leq 1+|t| \widehat{N}(|t|)$ and the point $t_{0}=\lim _{n \rightarrow \infty} 1 / \sqrt[n]{\widehat{N}_{n}}=\lim _{n \rightarrow \infty} 1 / \sqrt[n]{N_{n}}$ in 
convergent circle is the pole of the series $1+t \widehat{N}(t)$. By (26), $t_{0}$ is the minimum positive zero of the polynomial function $\beta(t)-\alpha(t)$; that is, $t_{0}$ is the minimum positive root of the model of the equation

$$
\beta(t)-\alpha(t)=0
$$

Substituting $t_{0}$ into (9), we obtain a formula of topological entropy of the map:

$$
h_{f}=-\ln t_{0}
$$

Example 6. Calculate the topological entropy of the map:

$$
g(x)= \begin{cases}1-\sqrt{2} x, & 0<x \leq 1 \\ -1-\sqrt{2} x, & -1 \leq x<0 .\end{cases}
$$

$\beta=(1,-1,-1,1,-1,1, \ldots)$ is an eventually periodic sequence; then $\beta(t)=1-t-t^{2} /(1+t)$. By the symmetry of the map $g(x)$, we have $\alpha=-\beta$, Substituting the expressions of $\alpha(t)$ and $\beta(t)$ into (27), we obtain $2\left(1-t-t^{2} /(1+t)\right)=0$ and $t_{0}=1 / \sqrt{2}$. Thus, $h_{g}=-\ln \left|t_{0}\right|=\ln \sqrt{2}$.

\section{Conclusion}

For the dynamic systems described by differential equations, the Lorenz Poincaré map is a mostly used method to study the structure of strange attractors of the systems. The dynamical behavior of the Lorenz-type attractor can be described by one-dimensional Lorenz-type map. The topological entropy of the Lorenz map can be calculated by using the symbol sequence of the boundary points of the invariant interval, and we can know the system is chaos or not.

\section{Competing Interests}

The author declares that there are no competing interests regarding the publication of this paper.

\section{References}

[1] V. S. Afraimovich, V. V. Bykov, and L. P. Shil'nikov, "On structurally unstable attracting limit sets of Lorenz attractor type," Transactions of the Moscow Mathematical Society, vol. 44, no. 2 , pp. 153-216, 1982.

[2] J. Guckenheimer and R. Williams, "Structural stability of the Lorenz attractor," Publ. Math. IEHS, vol. 50, pp. 59-72, 1980.

[3] R. Williams, “The structure of Lorenz attractors," Publications Mathématiques de l'IHÉS, vol. 50, pp. 101-152, 1979.

[4] J. Guckenheimer, "A strange, strange attractor," in Hopf Bifurcation and Its Applications, pp. 368-381, Springer, Berlin, Germany, 1976.

[5] A. L. Shil'nikov, "On bifurcations of the Lorenz attractor in the Shimizu-Morioka model," Physica D, vol. 62, no. 1-4, pp. 338346, 1993.

[6] A. L. Shil'nikov, "Bifurcation and chaos in the Shimizu-Morioka model system," Selecta Mathematica Sovietica, vol. 10, no. 2, pp. 455-568, 1991.
[7] V. Afraimovich and S.-B. Hsu, Lectures on Chaotic Dynamical Systems, American Mathematical Society and International Press, 2003.

[8] W. de Melo and S. van Strien, One-Dimensional Dynamics, Springer, Berlin, Germany, 1993. 


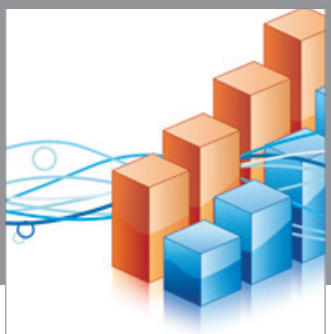

Advances in

Operations Research

vatem alat4

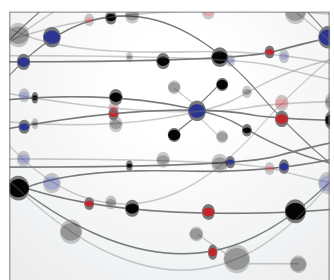

\section{The Scientific} World Journal
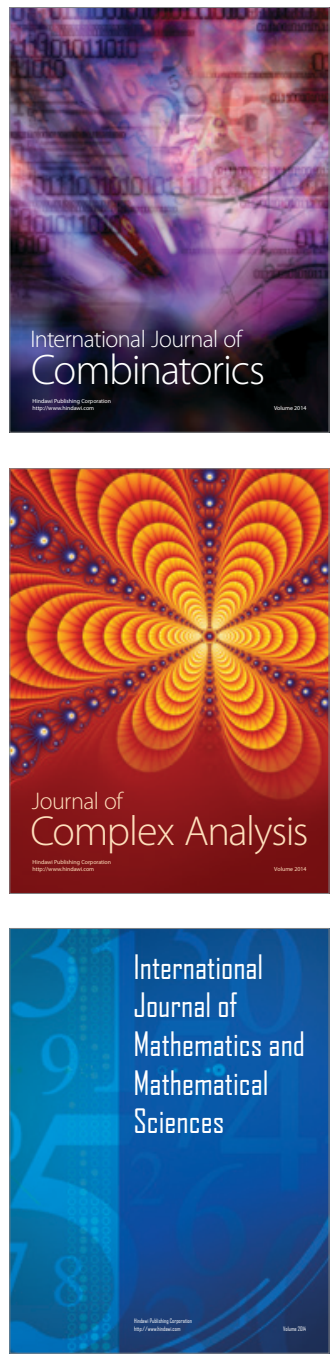
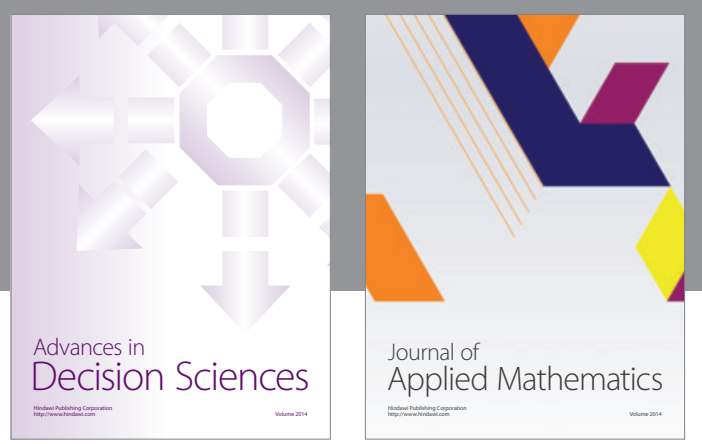

Algebra

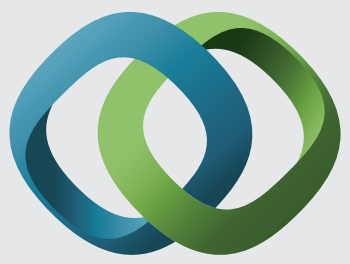

\section{Hindawi}

Submit your manuscripts at

http://www.hindawi.com
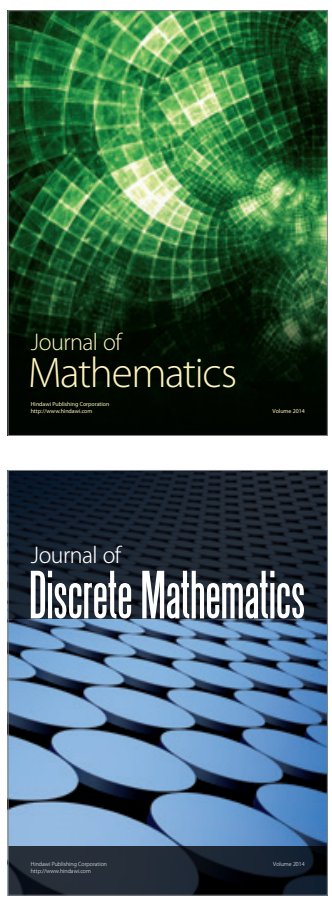

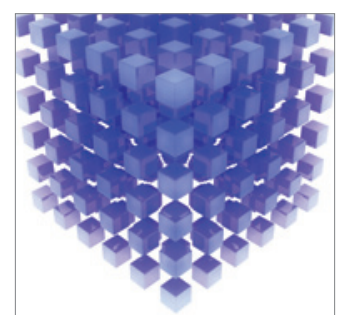

Mathematical Problems in Engineering
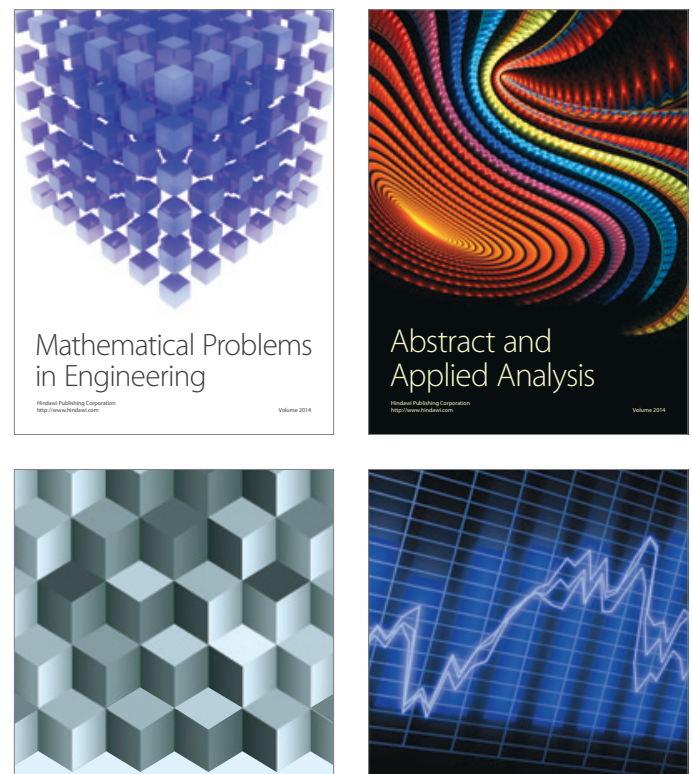

Journal of

Function Spaces

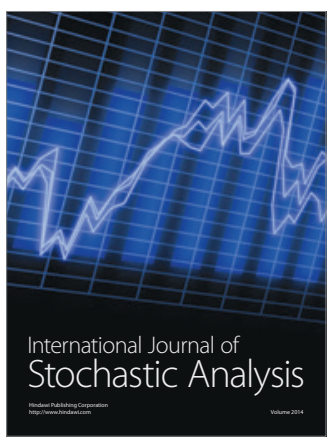

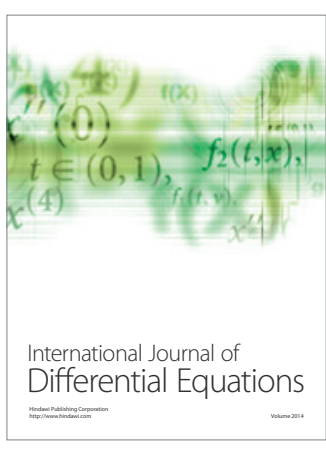
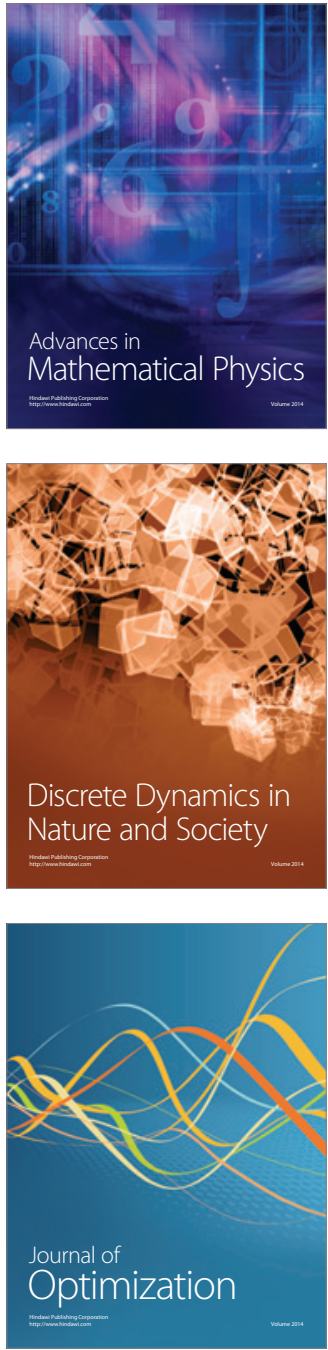